\title{
Structural Consequences of the Villin Headpiece Interaction with a Carbon Nitride Polyaniline $\left(\mathrm{C}_{3} \mathrm{~N}\right)$ Nanosheet
}

\author{
Zonglin $\mathrm{Gu}^{1}$, Jose Manuel Perez-Aguilar ${ }^{2}$, Ruhong Zhou ${ }^{1,3} *$ \\ ${ }^{1}$ Institute of Quantitative Biology, Zhejiang University, Hangzhou 310027, China \\ ${ }^{2}$ School of Chemical Sciences, Meritorious Autonomous University of Puebla (BUAP), \\ University City, Puebla 72570, Mexico \\ ${ }^{2}$ Department of Chemistry, Columbia University, New York, NY 10027, Unites States \\ *All correspondences should be addressed to: rhzhou@ zju.edu.cn
}

\begin{abstract}
Carbon nitride polyaniline $\left(\mathrm{C}_{3} \mathrm{~N}\right)$ nanosheets shared a similar structure with graphene and have been utilized in biomedical applications since its recent successful synthesis. However, limited information was known about the interaction of this next-generation nanomaterial with biomolecules, which might hamper its applications in living tissues. Here, by using all-atom molecular dynamics (MD) simulations, we investigated the interaction between a $\mathrm{C}_{3} \mathrm{~N}$ nanosheet and the prototypical protein villin headpiece (HP35), in order to identify the mechanistic determinants of such interaction; this knowledge will provide guidelines about $\mathrm{C}_{3} \mathrm{~N}$ 's biocompatibility. Our MD simulations revealed that the $\mathrm{C}_{3} \mathrm{~N}$-based nanomaterial caused the partial denaturation of HP35 once the protein was bound on its surface. That is, upon adsorption, we observed the loss of the protein's interior hydrogen bonds and the native contacts, which were related with unwinding events in the protein's helices. The protein/ $\mathrm{C}_{3} \mathrm{~N}$ nanosheet interacting process was dominated by vdW contributions to the energy and the stepwise changes observed in the values of this energy term suggested a gradual unfolding pattern of HP35 during the absorption event. Furthermore, we also found
\end{abstract}


that the interaction energy showed a linear correlation with the native Q ratio of HP35, suggesting that the degree of HP35 unfolding was linearly time-dependent to the interaction energy. Our findings shed light on the underlying molecular mechanism of the potential consequences of $\mathrm{C}_{3} \mathrm{~N}$-based nanostructures to proteins, which might delineate the future applications of these nanomaterials in biomedicine.

\section{INTRODUCTION}

The rapid development of a wide variety of nanomaterials have propelled their applications in many scientific areas, including energy conversion, generation of electronic and optical devices, and medicine. ${ }^{1-9}$ Among these and since their discovery, ${ }^{10-12}$ carbon based nanomaterials (CBNs) have received significant attention based on their remarkable mechanical, optical, and electrical properties ${ }^{4,13-14}$, and particularly in the biomedical field, ${ }^{15-16}$ where they have been applied as possible drug and gene delivery carriers, ${ }^{17}$ in optical imaging devices,${ }^{18}$ and in different medical treatments (nanotherapeutics). ${ }^{19-21}$ Nonetheless, special attention must be considered during the incorporation of such nanostructures into biological systems, due to potential deleterious effects of these exogenous materials on living cells. When integrated with living organisms, the nanomaterials could either disrupt different cellular components (e.g., cell membrane, proteins, and nucleic acids) and signaling pathways or display little effect in the normal function of the cell and living tissues (i.e., biocompatibility). Regardless of the particular outcome, cytotoxic or biocompatible, the exogenous nanomaterials will encounter a variety of biochemical milieus and consequently, different cellular entities which strengthened the significance to investigate the specifics of the nanomaterial-biomolecule interactions. For example, direct interaction of $\mathrm{CBN}$ with proteins or nucleic acids will generate toxicity via the denaturation of the biomolecular native structures (e.g., loss of secondary and/or tertiary structures). ${ }^{22-23}$ Also, by occupying the active site of a receptor and hence inhibiting the native donor-receptor interaction, carbon nanotubes (CNT) can interrupt the normal signal transmission via a non-unfolding pathway. ${ }^{24}$ Moreover, graphene nanosheets can penetrate into and extract large amounts of 
phospholipids from cell membranes due to the strong dispersion interactions resulting in a significant toxicity by these nanostructures - this graphene's property suggested the use of this nanomaterial as a possible antibacterial agent. ${ }^{25}$ Lastly, due to their strong surface attraction and influence on the structure and function of biomolecules, CBNs often had to be functionalized prior to their utilization in biomedical applications to enhance biocompatibility—usually by surface modifications with polyethylene glycol or serum proteins. ${ }^{26-27}$

Beyond CBNs, transition-metal dichalcogenides (TMD) nanostructures, particularly those based on molybdenum disulfide $\left(\mathrm{MoS}_{2}\right),{ }^{28}$ have recently emerged as another promising nanomaterial. Interestingly, $\mathrm{MoS}_{2}$-based nanomaterials shared similar physicochemical characteristics with CBNs, and thus, replication of the success application of $\mathrm{CBN}$ in the biomedical field by $\mathrm{MoS}_{2}$-based nanostructures was anticipated. Recent studies have demonstrated the application of $\mathrm{MoS}_{2}$-based nanomaterials as antibacterial and antifungal agents, ${ }^{29}$ biosensors (based on their unique direct band gap), ${ }^{30-31}$ photothermal and chemotherapeutic agents (high near-infrared absorbance and extensive specific surface area), ${ }^{32-33}$ as well as contrast agents in X-ray tomography imaging. ${ }^{33}$ Given the extensive usages of $\mathrm{MoS}_{2}$ nanostructures in biomedicine, our previous investigations exposed the direct interaction of $\mathrm{MoS}_{2}$ and biomolecules and found that pristine $\mathrm{MoS}_{2}$ had a non-negligible impact on the protein structure due to its strong attraction, ${ }^{34-37}$ implying the potential toxicity of $\mathrm{MoS}_{2}$-based nanomaterials.

More recently, the successful synthesis of carbon nitride compounds, such as $\mathrm{C}_{2} \mathrm{~N},{ }^{38}$ g- $_{3} \mathrm{~N}_{4}$ (graphitic carbon nitride that includes those materials based on heptazine and triazine units), polytriazine imide (PTI), ${ }^{39}$ as well as polyaniline $\left(\mathrm{C}_{3} \mathrm{~N}\right),{ }^{40-41}$ provided a novel class of nanomaterials for potential biomedical applications. In this regard, the ultrathin $\mathrm{g}-\mathrm{C}_{3} \mathrm{~N}_{4}$ nanosheet prepared by a "green" liquid exfoliation route, has been applied as bioimaging candidate with various advantages, including, enhanced intrinsic photoabsorption and photoresponse, high stability, good biocompatibility, and nontoxicity. ${ }^{42}$ Moreover, a g- $\mathrm{C}_{3} \mathrm{~N}_{4}$-sensitized $\mathrm{TiO}_{2}$ nanotube layer system was produced as visible-light activated efficient 
metal-free antimicrobial platform. ${ }^{43}$ In addition, the folic acid modified $\mathrm{C}_{3} \mathrm{~N}$ dots were selectively endocytosed by and killed tumor cells. ${ }^{44}$ Similarly, $2 \mathrm{D} \mathrm{C}_{3} \mathrm{~N}$ could be utilized as biosensor. ${ }^{45}$ Despite their diverse applications in biological systems, so far there are very limited studies or evidences to disclose the interaction dynamics and/or potential molecular mechanism of biomolecules binding to carbon nitride compounds. Our recent work probed the adhesion of the prototypical protein villin headpiece (HP35) to a $\mathrm{C}_{2} \mathrm{~N}$ nanosheet and found that the protein's structural integrity was well retained with weaken transverse migration on the $\mathrm{C}_{2} \mathrm{~N}$ surface. ${ }^{46}$ Herein, we extended the molecular characterization of carbon nitride compounds to include $\mathrm{C}_{3} \mathrm{~N}$ (polyaniline)-based nanostructures. By employing a 2-dimensional $\mathrm{C}_{3} \mathrm{~N}$ nanosheet and HP35, we explored the structural consequences at the nano-bio interface of this system-detailed knowledge of the interaction dynamics and molecular mechanism, which was fundamental for its potential applications in biomedicine. Our results revealed that the HP35 protein partially unfolded once absorbed onto the $\mathrm{C}_{3} \mathrm{~N}$ nanosheet, which suggested that this carbon-nitrogen-based nanomaterial might have potential influence in modifying biomolecular structures and consequently, potential toxicity to living tissues.

\section{RESULTS}

The 2-dimensional graphene-like $\mathrm{C}_{3} \mathrm{~N}$ (also named as polyaniline) nanosheet consisted of the basic unit formed by a benzene ring surrounded by six nitrogen atoms, which was polymerized by anilines ${ }^{40-41}$ (as shown in Fig. 1a). HP35, a 35-residue subdomain of the villin headpiece (as shown in Fig. 1b), was a small ultrafast folding protein that has been extensively studied by experiments, theory, and simulations. ${ }^{47-48}$ Herein, we employed the well-studied HP35 protein as model system to explore the possible consequences of the presence of the $\mathrm{C}_{3} \mathrm{~N}$ nanosheet to biomolecule. Two systems (a typical system configuration was displayed in Fig. 1c) were investigated by three independent 1000-ns-length atomistic MD simulations.

Figure 2a illustrated the relation between the protein $/ \mathrm{C}_{3} \mathrm{~N}$ nanosheet interaction energy and the changes in the native protein structure. Notably, stronger interactions 
between the HP35 and $\mathrm{C}_{3} \mathrm{~N}$ were directly related with larger structural alterations of the protein structure. Moreover, the loss of the HP35 helical content of its secondary elements (helices $\alpha 1, \alpha 2$, and $\alpha 3$ ) occurred in two of the cases, with remaining helical content values of $40.2 \pm 13.9 \%$ and $61.5 \pm 3.8 \%$. The representative structures of HP35 in the insets of Fig. 2a, exemplified conformations where the complete loss of helix $\alpha 1$ and helix $\alpha 2$ was observed. The contact probabilities (Fig. 2b) of each residue bound to $\mathrm{C}_{3} \mathrm{~N}$ along the simulations showed that the residues L1, S2, D3, E4, S15, N19, L27, and F35, were particularly important in mediating the absorption of HP35 onto the surface. Note that the F35 residue at the C-terminus, presented the largest contact probability, up to $78.9 \%$. Detailed analysis demonstrated that F35 formed a face-to-face pattern with the $\mathrm{C}_{3} \mathrm{~N}$ surface, yielding a direct interaction energy of $-16.4 \pm 1.0 \mathrm{kcal} / \mathrm{mol}$ (Fig. 2c), which suggested its relevance in the binding process-equivalent interactions were observed in the case of HP35 and graphene. ${ }^{22}$

Next, we performed a dihedral principal components analysis (dPCA) of HP35 bound to $\mathrm{C}_{3} \mathrm{~N}$ (Fig. 3). Notably, from 7 binding protein conformations on the $\mathrm{C}_{3} \mathrm{~N}$ surface extracted from the dPCA plot (namely, conformations i to vii), in 5 of them, HP35 seemingly maintained its structural integrity. However, in two conformations, partial denaturation was observed (conformation ii and iii), that is, the secondary structure of helix $\alpha 2$ was lost in conformation ii while the secondary structure of helix $\alpha 1$ was lost in conformation iii (also see Fig. 2a). This finding evidenced the potential denaturation capacity of the $\mathrm{C}_{3} \mathrm{~N}$ nanostructures to the HP35 protein.

Then, detailed analyses were further conducted to quantitatively measure structural changes of HP35 in binding to the $\mathrm{C}_{3} \mathrm{~N}$ surface by selecting a representative trajectory (Fig. 4). A root-mean-square deviation (RMSD) calculation of the HP35 protein reflected a substantial increment from its initial value during the $1000 \mathrm{~ns}$ simulation time, revealing the significant influence of the $\mathrm{C}_{3} \mathrm{~N}$ surface (Fig. 4a). Moreover, the number of internal hydrogen bonds in HP35 declined from $\sim 30$ to $\sim 14$ and the native $\mathrm{Q}$ value was reduced to $57 \%$ (Fig. 4c). The time-dependent $\mathrm{Q}$ change of each residue showed that most residues lose their native contacts with nearby residues (Fig. 4c), particularly the residues V9, G11, and F35 (with $\Delta \mathrm{Q}=1.0$ ). Furthermore, a map with 
the time-evolution secondary structure content illustrated the entire loss of helix $\alpha 1$ and the partial loss of helix $\alpha 3$ (Fig. 4d). Combined, the quantitative calculations further confirmed the significant depletion of the structural integrity of HP35 upon binding to the $\mathrm{C}_{3} \mathrm{~N}$ nanosheet.

To trace the binding kinetics of the system, the relevant aforementioned conformations were depicted based on the change in contact number and in interaction energy (Fig. 5). Both, the contact number value and vdW interaction energy exhibited stepwise changes, implying that the HP35 discretely and gradually unfolded and bound to the $\mathrm{C}_{3} \mathrm{~N}$ nanosheet, respectively. More importantly, the vdW contributions dominated the interaction throughout the adsorption process. Mechanistically, at $\mathrm{t}=13 \mathrm{~ns}$, the HP35 established direct contact via the helix $\alpha 1$ as the contact number value rose to $\sim 43$ and the vdW energy declined to $-53 \mathrm{kcal} / \mathrm{mol}$. After $212 \mathrm{~ns}$ simulation ( $\mathrm{t}=225 \mathrm{~ns})$, the orientation of HP35 on the surface slightly changed via a rotation, which bring the $\mathrm{F} 35$ residue at the $\mathrm{C}$-terminus in contact with the $\mathrm{C}_{3} \mathrm{~N}$ nanosheet. This last event was accompanied by an increment in the contact number value, $\sim 63$, and a decrease in the vdW energy, $-82.4 \mathrm{kcal} / \mathrm{mol}$. At this time point, the native structure of HP35 was well maintained without any obvious structural change. However shortly after, at $t=291$ ns, the HP35 lay on the $\mathrm{C}_{3} \mathrm{~N}$ surface with its three helices all contacting to $\mathrm{C}_{3} \mathrm{~N}$ nanosheet in a parallel way with the concomitant loss of the HP35 native structure (exposure and disruption of its hydrophobic core). Meanwhile, the aromatic tryptophan residue, W23, in helix $\alpha 3$ was also positioned in proximity of the $C_{3} N$ surface. Lastly, after $t=400 \mathrm{~ns}$, we observed a significant loss in the protein helical content attributed to the complete and partial unwinding of helix $\alpha 1$ and helix $\alpha 3$, respectively. Additionally, direct interaction of several important residues on helix $\alpha 1-\mathrm{L} 1, \mathrm{~K} 7, \mathrm{~A} 8$ and F10 — with the $\mathrm{C}_{3} \mathrm{~N}$ nanosheet was established and maintained for the rest of the simulation. Remarkably, we observed a central role of aromatic and basic residues in the surface-binding event, which was also identified in the protein/graphene interaction. $^{49-50}$

Finally, we probed the correlation between the interaction energy and native Q ratio 
in the two representative trajectories where the HP35 unfolding processes were observed, conformation ii and iii (Fig. 6 and S1). Notably, the interaction energy and native $\mathrm{Q}$ ratio displayed a time-dependent linear correlation, implying that the unfolding of HP35 on the $\mathrm{C}_{3} \mathrm{~N}$ surface had a highly linear correlation with respect to the interaction energy.

\section{CONCLUSION}

In summary, we explored the potential influence of a $\mathrm{C}_{3} \mathrm{~N}$ nanosheet into the structure of the HP35 protein. Our results showed that HP35 was largely denatured upon adsorption to the $\mathrm{C}_{3} \mathrm{~N}$ nanosheet. Statistical contact probability demonstrated that the C-terminal F35 residue was a central residue during the surface-binding event. dPCA suggested that in two out of the seven conformations explored by HP35, a significant reduction in the structural integrity of the protein occurred. Further structural analyses quantitatively described the loss in the number of interior hydrogen bonds and native contacts. The HP35 bound to $\mathrm{C}_{3} \mathrm{~N}$ surface in a stepwise fashion, which was driven by the vdW energy dominant contribution. The aromatic and basic residues exhibited a critical role during the adsorption and unfolding processes. Moreover, the interaction energy and native $\mathrm{Q}$ ratio displayed a time-dependent linear correlation. Our results confirmed the structural influence of the $\mathrm{C}_{3} \mathrm{~N}$ nanosheet to protein, which thereby implied a potential toxicity of $\mathrm{C}_{3} \mathrm{~N}$ nanomaterial and the need to functionalize its surface to increase biocompatibility for biomedical application.

\section{METHODS}

The $\mathrm{C}_{3} \mathrm{~N}$ nanosheet monolayer, treated as benzene rings surrounded and connected by six nitrogen atoms, was displayed in Fig. 1a. The $\mathrm{C}_{3} \mathrm{~N}$ model utilized in this study consisted of 1,152 carbon and 384 nitrogen atoms with a surface dimension of $5.83 \times 6.73 \mathrm{~nm}^{2}$. The 35 -residue chicken villin headpiece subdomain protein (HP35, PDB code: 1 YRF ${ }^{51}$ was selected because of its general properties associated with common globular proteins despite its small size. ${ }^{52-55}$ The native structure of HP35 contained a globular bundle of three $\alpha$-helices, namely helix $\alpha 1$, helix $\alpha 2$ and helix 
$\alpha 3$, from N-terminus to C-terminus, as shown in Fig. 1b. The HP35 was originally placed above the $\mathrm{C}_{3} \mathrm{~N}$ nanosheet with their initial distance at $1.5 \mathrm{~nm}$. In this study, we built two systems (one representation was illustrated in Fig. 1c) in which the HP35 was rotated for $180^{\circ}$ yielding two different orientations relative to the $\mathrm{C}_{3} \mathrm{~N}$ surface. The system boxes were set to $5.83 \times 6.73 \times 4.92 \mathrm{~nm}^{3}$ and $5.83 \times 6.73 \times 4.90 \mathrm{~nm}^{3}$ containing 5,729 and 5,732 water molecules, respectively. In addition, $0.15 \mathrm{M} \mathrm{NaCl}$ were also dissolved in two systems to mimic physiological environment.

The simulations were carried out with the GROMACS software package (version 4.6.6 ${ }^{56}$ using the CHARMM36 force field. ${ }^{57}$ The force field for $\mathrm{C}_{3} \mathrm{~N}$ was obtained from a recent work. ${ }^{58}$ The VMD software ${ }^{59}$ was used to analyze and visualize the simulation results. The TIP3P water model ${ }^{60}$ was adopted to treat water molecules. Following similar protocols in our previous studies ${ }^{61-67}$, the temperature were maintained at $300 \mathrm{~K}$ using v-rescale thermostat ${ }^{68}$ and pressure was kept at $1 \mathrm{~atm}$ using semiisotropic Berendsen barostat ${ }^{69}$ (only applied at $\mathrm{Z}$ direction perpendicular to the $\mathrm{C}_{3} \mathrm{~N}$ nanosheet). To avoid the "artificial collapsing" of nanosheets with their mirror images due to the limited size of simulation box (which was due to the limited computational resources), the $\mathrm{C}_{3} \mathrm{~N}$ nanosheet was fixed throughout the simulation

process. The long-range electrostatic interactions were treated with PME method, ${ }^{70}$ and the van der Waals (vdW) interactions were calculated with a cutoff distance of 1.2 $\mathrm{nm}$. All solute bonds associated with hydrogen atoms were maintained constant at their equilibrium values with the LINCS algorithm, ${ }^{71}$ and water geometry was also constrained using the SETTLE algorithm. ${ }^{72}$ During the production runs, a time step of 2.0 fs was used, and data were collected every 50 ps. Each system ran for three 1000 ns trajectories. The total aggregated simulation time was about $6 \mu \mathrm{s}$.

\section{ACKNOWLEDGEMENT}

We thank Zhi He and Jianxiang Huang for help with the manuscript. This work is partially supported by the National Natural Science Foundation of China (Grants 11574224 and U1967217) and China Postdoctoral Science Foundation (Grant 
2019M652069 and 2019T120506). R.Z. also acknowledges the financial support from

W. M. Keck Foundation (Grant award 2019-2022).

\section{REFERENCES}

1. Elimelech, M.; Phillip, W. A., The future of seawater desalination: Energy, technology, and the environment. Science 2011, 333 (6043), 712-717.

2. Shannon, M. A.; Bohn, P. W.; Elimelech, M.; Georgiadis, J. G.; Marinas, B. J.; Mayes, A. M., Science and technology for water purification in the coming decades. Nature 2008, 452 (7185), 301-310.

3. Liu, M.; Yin, X.; Zhang, X., Double-layer graphene optical modulator. Nano Lett. 2012, 12 (3), $1482-1485$.

4. Geim, A. K., Graphene: Status and prospects. Science 2009, 324 (5934), 1530-1534.

5. Chen, P.; Wu, X.; Lin, J.; Tan, K., High h2 uptake by alkali-doped carbon nanotubes under ambient pressure and moderate temperatures. Science 1999, 285 (5424), 91-93.

6. Guo, Z.; Wu, H.; Srinivas, G.; Zhou, Y.; Xiang, S.; Chen, Z.; Yang, Y.; Zhou, W.; O'Keeffe, M.; Chen, B., A metal-organic framework with optimized open metal sites and pore spaces for high methane storage at room temperature. Angew. Chem. Int. Edit. 2011, 50 (14), 3178-3181.

7. Bolotin, K. I.; Sikes, K. J.; Jiang, Z.; Klima, M.; Fudenberg, G.; Hone, J.; Kim, P.; Stormer, H. L., Ultrahigh electron mobility in suspended graphene. Solid State Commun. 2008, 146 (9-10), 351-355.

8. Yang, K.; Zhang, S.; Zhang, G.; Sun, X.; Lee, S.-T.; Liu, Z., Graphene in mice: Ultrahigh in vivo tumor uptake and efficient photothermal therapy. Nano Lett. 2010, 10 (9), 3318-3323.

9. Mao, H. Y.; Laurent, S.; Chen, W.; Akhavan, O.; Imani, M.; Ashkarran, A. A.; Mahmoudi, M., Graphene: Promises, facts, opportunities, and challenges in nanomedicine. Chem. Rev. 2013, 113 (5), 3407-3424.

10. Novoselov, K. S.; Geim, A. K.; Morozov, S.; Jiang, D.; Zhang, Y.; Dubonos, S. a.; Grigorieva, I.; Firsov, A., Electric field effect in atomically thin carbon films. Science 2004, 306 (5696), 666-669.

11. Kroto, H. W.; Heath, J. R.; Obrien, S. C.; Curl, R. F.; Smalley, R. E., C60 : Buckminsterfullerene. Nature 1985, 318 (6042), 162-163.

12. lijima, S., Helical microtubes of graphitic carbon. Nature 1991, 354 (6348), 56-58.

13. Feng, L.; Liu, Z., Graphene in biomedicine: Opportunities and challenges. Nanomedicine 2011, 6 (2), 317-324.

14. Sanchez, V. C.; Jachak, A.; Hurt, R. H.; Kane, A. B., Biological interactions of graphene-family nanomaterials: An interdisciplinary review. Chemical Research in Toxicology 2012, 25 (1), 15-34.

15. Lee, J. S.; Joung, H.-A.; Kim, M.-G.; Park, C. B., Graphene-based chemiluminescence resonance energy transfer for homogeneous immunoassay. ACS Nano 2012, 6 (4), 2978-2983.

16. Cha, C.; Shin, S. R.; Annabi, N.; Dokmeci, M. R.; Khademhosseini, A., Carbon-based nanomaterials: Multifunctional materials for biomedical engineering. ACS Nano 2013, 7 (4), 2891-2897.

17. Bao, H.; Pan, Y.; Ping, Y.; Sahoo, N. G.; Wu, T.; Li, L.; Li, J.; Gan, L. H., Chitosan-functionalized graphene oxide as a nanocarrier for drug and gene delivery. Small 2011, 7 (11), 1569-1578.

18. Li, B.; Cheng, Y.; Liu, J.; Yi, C.; Brown, A. S.; Yuan, H.; Tuan, V.-D.; Fischer, M. C.; Warren, W. S., Direct optical imaging of graphene in vitro by nonlinear femtosecond laser spectral reshaping. Nano Lett. 2012, 12 (11), 5936-5940.

19. Li, M.; Yang, X.; Ren, J.; Qu, K.; Qu, X., Using graphene oxide high near-infrared absorbance for 
photothermal treatment of alzheimer's disease. Adv. Mater. 2012, 24 (13), 1722-1728.

20. Yang, K.; Wan, J.; Zhang, S.; Tian, B.; Zhang, Y.; Liu, Z., The influence of surface chemistry and size of nanoscale graphene oxide on photothermal therapy of cancer using ultra-low laser power. Biomaterials 2012, 33 (7), 2206-2214.

21. Yang, Z.; Kang, S.-g.; Zhou, R., Nanomedicine: De novo design of nanodrugs. Nanoscale 2014, 6 (2), 663-677.

22. Zuo, G.; Zhou, X.; Huang, Q.; Fang, H.; Zhou, R., Adsorption of villin headpiece onto graphene, carbon nanotube, and c60: Effect of contacting surface curvatures on binding affinity. J. Phys. Chem. $C$ 2011, 115 (47), 23323-23328.

23. Zhao, X., Self-assembly of DNA segments on graphene and carbon nanotube arrays in aqueous solution: A molecular simulation study. J. Phys. Chem. C 2011, 115 (14), 6181-6189.

24. Zuo, G.; Huang, Q.; Wei, G.; Zhou, R.; Fang, H., Plugging into proteins: Poisoning protein function by a hydrophobic nanoparticle. ACS Nano 2010, 4 (12), 7508-14.

25. Tu, Y.; Lv, M.; Xiu, P.; Huynh, T.; Zhang, M.; Castelli, M.; Liu, Z.; Huang, Q.; Fan, C.; Fang, H., et al., Destructive extraction of phospholipids from escherichia coli membranes by graphene nanosheets. Nat. Nanotechnol. 2013, 8 (8), 594-601.

26. Sacchetti, C.; Motamedchaboki, K.; Magrini, A.; Palmieri, G.; Mattei, M.; Bernardini, S.; Rosato, N.; Bottini, N.; Bottini, M., Surface polyethylene glycol conformation influences the protein corona of polyethylene glycol-modified single-walled carbon nanotubes: Potential implications on biological performance. ACS Nano 2013, 7 (3), 1974-1989.

27. Ge, C.; Du, J.; Zhao, L.; Wang, L.; Liu, Y.; Li, D.; Yang, Y.; Zhou, R.; Zhao, Y.; Chai, Z., et al., Binding of blood proteins to carbon nanotubes reduces cytotoxicity. P. Natl. Acad. Sci. USA 2011, 108 (41), 16968-16973.

28. Coleman, J. N.; Lotya, M.; O'Neill, A.; Bergin, S. D.; King, P. J.; Khan, U.; Young, K.; Gaucher, A.; De, S.; Smith, R. J., et al., Two-dimensional nanosheets produced by liquid exfoliation of layered materials. Science 2011, 331 (6017), 568-571.

29. Yang, X.; Li, J.; Liang, T.; Ma, C.; Zhang, Y.; Chen, H.; Hanagata, N.; Su, H.; Xu, M., Antibacterial activity of two-dimensional mos2 sheets. Nanoscale 2014, 6 (17), 10126-10133.

30. Wang, L.; Wang, Y.; Wong, J. I.; Palacios, T.; Kong, J.; Yang, H. Y., Functionalized mos2 nanosheet-based field-effect biosensor for label-free sensitive detection of cancer marker proteins in solution. Small 2014, 10 (6), 1101-1105.

31. Zhu, C.; Zeng, Z.; Li, H.; Li, F.; Fan, C.; Zhang, H., Single-layer mos2-based nanoprobes for homogeneous detection of biomolecules. J. Am. Chem. Soc. 2013, 135 (16), 5998-6001.

32. Wang, S.; Li, K.; Chen, Y.; Chen, H.; Ma, M.; Feng, J.; Zhao, Q.; Shi, J., Biocompatible pegylated mos2 nanosheets: Controllable bottom-up synthesis and highly efficient photothermal regression of tumor. Biomaterials 2015, 39, 206-217.

33. Yin, W.; Yan, L.; Yu, J.; Tian, G.; Zhou, L.; Zheng, X.; Zhang, X.; Yong, Y.; Li, J.; Gu, Z., et al., High-throughput synthesis of single-layer mos 2 nanosheets as a near-infrared photothermal-triggered drug delivery for effective cancer therapy. ACS Nano 2014, 8 (7), 6922-6933.

34. Gu, Z.; Li, W.; Hong, L.; Zhou, R., Exploring biological effects of mos2 nanosheets on native structures of $\alpha$-helical peptides. J. Chem. Phys. 2016, 144 (17), 175103.

35. Gu, Z.; Yang, Z.; Kang, S.-G.; Yang, J. R.; Luo, J.; Zhou, R., Robust denaturation of villin headpiece by mos2 nanosheet: Potential molecular origin of the nanotoxicity. Sci. Rep. 2016, 6, 28252.

36. Ling, Y.; Gu, Z.; Kang, S.-g.; Luo, J.; Zhou, R., Structural damage of a beta-sheet protein upon 
adsorption onto molybdenum disulfide nanotubes. J. Phys. Chem. C 2016, 120 (12), 6796-6803.

37. Gu, Z.; Plant, L. D.; Meng, X.; Perez-Aguilar, J. M.; Wang, Z.; Dong, M.; Logothetis, D. E.; Zhou, R., Exploring the nanotoxicology of mos2: A study on the interaction of mos2 nanoflake and k+ channels. ACS Nano 2018, 12 (1), 705-717.

38. Mahmood, J.; Lee, E. K.; Jung, M.; Shin, D.; Jeon, I.-Y.; Jung, S.-M.; Choi, H.-J.; Seo, J.-M.; Bae, S.-Y.; Sohn, S.-D., et al., Nitrogenated holey two-dimensional structures. Nat. Commun. 2015, 6, 6486.

39. Mansor, N.; Miller, T. S.; Dedigama, I.; Jorge, A. B.; Jia, J.; Brazdova, V.; Mattevi, C.; Gibbs, C.; Hodgson, D.; Shearing, P. R., et al., Graphitic carbon nitride as a catalyst support in fuel cells and electrolyzers. Electrochim. Acta 2016, 222, 44-57.

40. Mahmood, J.; Lee, E. K.; Jung, M.; Shin, D.; Choi, H. J.; Seo, J. M.; Jung, S. M.; Kim, D.; Li, F.; Lah, M. S., et al., Two-dimensional polyaniline $(\mathrm{c} 3 \mathrm{n})$ from carbonized organic single crystals in solid state. $P$. Nati. Acad. Sci. USA 2016, 113 (27), 7414-7419.

41. Yang, S. W.; Li, W.; Ye, C. C.; Wang, G.; Tian, H.; Zhu, C.; He, P.; Ding, G. Q.; Xie, X. M.; Liu, Y., et al., C3n-a 2d crystalline, hole-free, tunable-narrow-bandgap semiconductor with ferromagnetic properties. Adv. Mater. 2017, 29 (16), 1605625.

42. Zhang, X. D.; Xie, X.; Wang, H.; Zhang, J. J.; Pan, B. C.; Xie, Y., Enhanced photoresponsive ultrathin graphitic-phase c3n4 nanosheets for bioimaging. J. Am. Chem. Soc. 2013, 135 (1), 18-21.

43. Xu, J.; Li, Y.; Zhou, X.; Li, Y.; Gao, Z.-D.; Song, Y.-Y.; Schmuki, P., Graphitic c3n4-sensitized tio2 nanotube layers: A visible-light activated efficient metal-free antimicrobial platform. Chem-Eur J. 2016, 22 (12), 3947-3951.

44. Li, J.; Yang, S.; Deng, Y.; Chai, P.; Yang, Y.; He, X.; Xie, X.; Kang, Z.; Ding, G.; Zhou, H., Emancipating

target-functionalized carbon dots from autophagy vesicles for a novel visualized tumor therapy. Adv. Funct. Mater. 2018, 28 (30), 1800881.

45. Rani, S.; Ray, S. J., Two-dimensional c3n based sub-10 nanometer biosensor. Phys. Chem. Chem. Phys. 2020, 22 (20), 11452-11459.

46. Li, B.; Li, W.; Perez-Aguilar, J. M.; Zhou, R., Mild binding of protein to $\mathrm{c} 2 \mathrm{n}$ monolayer reveals its suitable biocompatibility. Small 2017, 13 (12), 1603685.

47. Lei, H.; Duan, Y., Two-stage folding of hp-35 from ab initio simulations. J. Mol. Biol. 2007, 370 (1), 196-206.

48. Chung, J. K.; Thielges, M. C.; Fayer, M. D., Dynamics of the folded and unfolded villin headpiece (hp35) measured with ultrafast $2 \mathrm{~d}$ ir vibrational echo spectroscopy. P. Natl. Acad. Sci. USA 2011, 108 (9), 3578-3583.

49. Gu, Z. L.; Yang, Z. X.; Wang, L. L.; Zhou, H.; Jimenez-Cruz, C. A.; Zhou, R. H., The role of basic residues in the adsorption of blood proteins onto the graphene surface. Sci. Rep. 2015, 5, 10873.

50. Gu, Z.; Song, W.; Chen, S. H.; Li, B.; Li, W.; Zhou, R., Defect-assisted protein hp35 denaturation on graphene. Nanoscale 2019, 11 (41), 19362-19369.

51. Chiu, T. K.; Kubelka, J.; Herbst-Irmer, R.; Eaton, W. A.; Hofrichter, J.; Davies, D. R., High-resolution $x$-ray crystal structures of the villin headpiece subdomain, an ultrafast folding protein. P. Natl Acad. Sci. USA 2005, 102 (21), 7517-7522.

52. Snow, C. D.; Nguyen, N.; Pande, V. S.; Gruebele, M., Absolute comparison of simulated and experimental protein-folding dynamics. Nature 2002, 420 (6911), 102-106.

53. Shaw, D. E.; Maragakis, P.; Lindorff-Larsen, K.; Piana, S.; Dror, R. O.; Eastwood, M. P.; Bank, J. A.; Jumper, J. M.; Salmon, J. K.; Shan, Y., et al., Atomic-level characterization of the structural dynamics of 
proteins. Science 2010, 330 (6002), 341-346.

54. Lei, H.; Wu, C.; Liu, H.; Duan, Y., Folding free-energy landscape of villin headpiece subdomain from molecular dynamics simulations. P. Natl. Acad. Sci. USA 2007, 104 (12), 4925-4930.

55. Duan, Y.; Kollman, P. A., Pathways to a protein folding intermediate observed in a 1-microsecond simulation in aqueous solution. Science 1998, 282 (5389), 740-744.

56. Hess, B.; Kutzner, C.; van der Spoel, D.; Lindahl, E., Gromacs 4: Algorithms for highly efficient, load-balanced, and scalable molecular simulation. J. Chem. Theory Comput. 2008, 4 (3), 435-447.

57. Huang, J.; MacKerell Jr, A. D., Charmm36 all-atom additive protein force field: Validation based on comparison to nmr data. J. Comput. Chem. 2013, 34 (25), 2135-2145.

58. Deng, Y.; Wang, F. X.; Liu, Y.; Yang, Y. M.; Qu, Y. Y.; Zhao, M. W.; Mu, Y. G.; Li, W. F., Orientational DNA binding and directed transport on nanomaterial heterojunctions. Nanoscale 2020, 12 (8), 5217-5226.

59. Humphrey, W.; Dalke, A.; Schulten, K., Vmd: Visual molecular dynamics. J. Mol. Graph. Model. 1996, 14 (1), 33-38.

60. Jorgensen, W. L.; Chandrasekhar, J.; Madura, J. D.; Impey, R. W.; Klein, M. L., Comparison of simple potential functions for simulating liquid water. J. Chem. Phys. 1983, 79 (2), 926-935.

61. Ahmed, R.; Omidian, Z.; Giwa, A.; Cornwell, B.; Majety, N.; Bell, D. R.; Lee, S.; Zhang, H.; Michels, A.; Desiderio, S., et al., A public bcr present in a unique dual-receptor-expressing lymphocyte from type 1 diabetes patients encodes a potent t cell autoantigen. Cell 2019, 177 (6), 1583-1599 e16.

62. Luo, N.; Weber, J. K.; Wang, S.; Luan, B.; Yue, H.; Xi, X.; Du, J.; Yang, Z.; Wei, W.; Zhou, R., et al., Pegylated graphene oxide elicits strong immunological responses despite surface passivation. Nat Commun 2017, 8, 14537.

63. Fitch, B. G.; Rayshubskiy, A.; Eleftheriou, M.; J., C. T.; Giampaga, M.; Zhestkov, Y.; Pitman, M. C.; Suits, F.; Grossfield, A.; Pitera, J., et al., Blue matter: Strong scaling of molecular dynamics on blue gene/l. Springe Berlin Heidelberg: International Conference on Computational Science, 2006.

64. Kaminski, G. A.; Friesner, R. A.; Zhou, R., A computationally inexpensive modification of the point dipole electrostatic polarization model for molecular simulations. J. Comput. Chem. 2003, 24 (3), 267-76.

65. Li, J.; Liu, T.; Li, X.; Ye, L.; Chen, H.; Fang, H.; Wu, Z.; Zhou, R., Hydration and dewetting near graphite-ch(3) and graphite-cooh plates. J Phys Chem B 2005, 109 (28), 13639-48.

66. Chowell, D.; Morris, L. G. T.; Grigg, C. M.; Weber, J. K.; Samstein, R. M.; Makarov, V.; Kuo, F.; Kendall, S. M.; Requena, D.; Riaz, N., et al., Patient hla class i genotype influences cancer response to checkpoint blockade immunotherapy. Science 2018, 359 (6375), 582-587.

67. Zhou, R., Exploring the protein folding free energy landscape: Coupling replica exchange method with p3me/respa algorithm. J. Mol. Graph. Model. 2004, 22 (5), 451-63.

68. Bussi, G.; Donadio, D.; Parrinello, M., Canonical sampling through velocity rescaling. J. Chem. Phys. 2007, 126 (1), 014101.

69. Berendsen, H. J. C.; Postma, J. P. M.; Vangunsteren, W. F.; Dinola, A.; Haak, J. R., Molecular-dynamics with coupling to an external bath. J. Chem. Phys. 1984, 81 (8), 3684-3690.

70. Darden, T.; York, D.; Pedersen, L., Particle mesh ewald - an n.Log(n) method for ewald sums in large systems. J. Chem. Phys. 1993, 98 (12), 10089-10092.

71. Hess, B.; Bekker, H.; Berendsen, H. J. C.; Fraaije, J., Lincs: A linear constraint solver for molecular simulations. J. Comput. Chem. 1997, 18 (12), 1463-1472. 
bioRxiv preprint doi: https://doi.org/10.1101/2020.06.17.158402; this version posted June 19, 2020. The copyright holder for this preprint (which was not certified by peer review) is the author/funder. All rights reserved. No reuse allowed without permission.

72. Miyamoto, S.; Kollman, P. A., Settle - an analytical version of the shake and rattle algorithm for rigid water models. J. Comput. Chem. 1992, 13 (8), 952-962. 

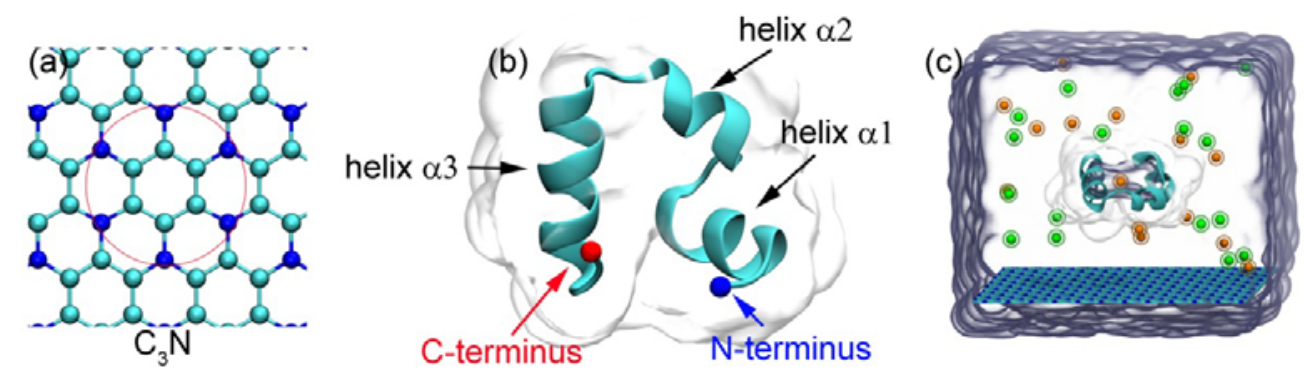

Figure 1. Configurations and simulation setup. (a) Structural conformation of $\mathrm{C}_{3} \mathrm{~N}$. Atoms in the red circle were the elementary unit of $\mathrm{C}_{3} \mathrm{~N}$ (i.e., polyaniline). Carbon and nitrogen atoms were represented as cyan and blue spheres, respectively. (b) Ribbon representation of the structure of HP35. The N- and C-termini were indicated by blue and red balls. The three helices composing the protein structure were also labeled as helix $\alpha 1$, helix $\alpha 2$ and helix $\alpha 3$ from the $\mathrm{N}$ - to the C-terminus. (c) Representation of the simulated systems investigated in this study. The HP35 protein was placed above the $\mathrm{C}_{3} \mathrm{~N}$ nanosheet and rotated by $180^{\circ}$ to form two different initial protein orientations. Their initial minimum distance was set to $1.5 \mathrm{~nm}$. $\mathrm{Na}^{+}$and $\mathrm{Cl}^{-}$were depicted as orange and green spheres. The $\mathrm{C}_{3} \mathrm{~N}$ sheet was shown with sticks. The water surface showed at the boundaries of the simulated periodic cell.
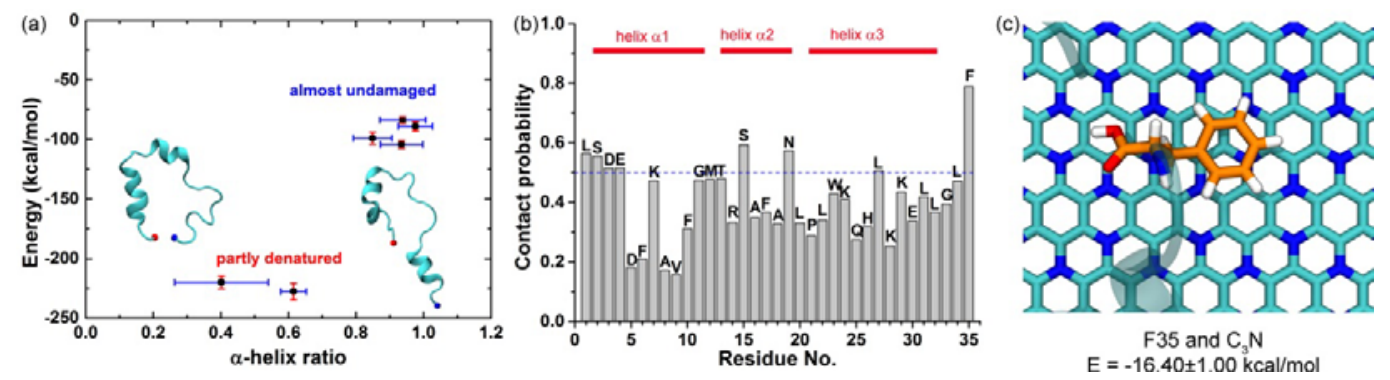

Figure 2. Energy/structure relation and residue contribution. (a) The relation between the interaction energy (including $\mathrm{vdW}$ and Coulomb energies between protein and $\mathrm{C}_{3} \mathrm{~N}$ ) and the secondary structural changes of HP35 collected from the last 50 ns of each trajectory. The inserted HP35 configurations were extracted from two "partly denatured" simulations (see main text). (b) Contact probability for each residue to the $\mathrm{C}_{3} \mathrm{~N}$ surface computed by all trajectories. A residue contact was considered if any heavy atom (non-hydrogen atom) of one residue had a distance to the $\mathrm{C}_{3} \mathrm{~N}$ surface smaller than $6.0 \AA$. The F35 residue exhibited the largest contact value, which 
was an indication of its central role in the absorption event. (c) Illustration of the interactions observed for $\mathrm{F} 35$ onto the $\mathrm{C}_{3} \mathrm{~N}$ sheet selected from a representative trajectory. The mean interaction energy between $\mathrm{F} 35$ and $\mathrm{C}_{3} \mathrm{~N}$ was calculated from the last $50 \mathrm{~ns}$.

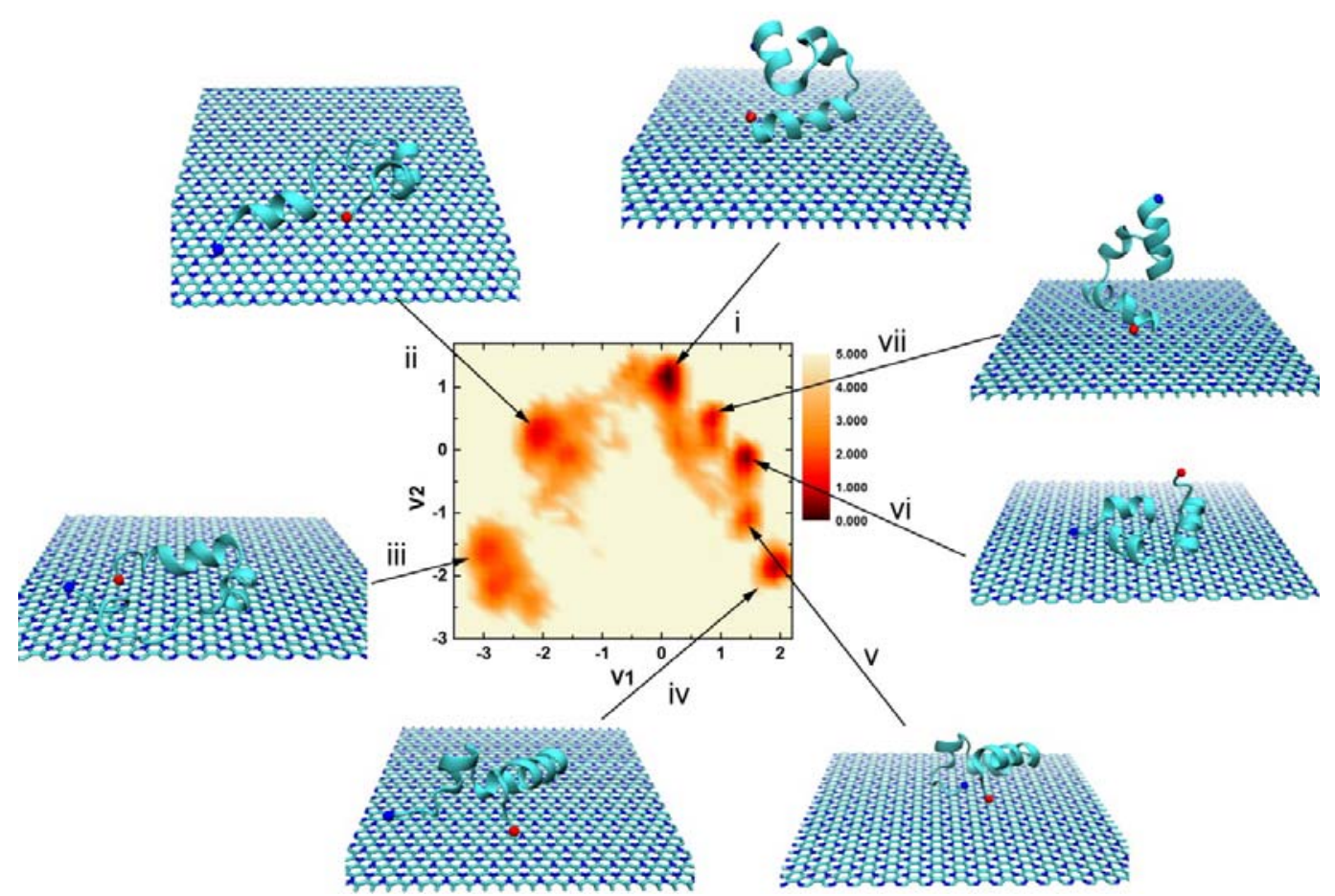

Figure 3. Dihedral principal components analysis (dPCA) of HP35. Projection of the free energy surface for the interaction of HP35 with the $\mathrm{C}_{3} \mathrm{~N}$ nanosheet in terms of the two lowest eigenvectors from the dPCA, obtained from the 6 independent 1000 ns trajectories. The color scale bar was in unit of kcal/mol. The key snapshots (including the HP35 and $\mathrm{C}_{3} \mathrm{~N}$ nanosheet) were plotted based on the free energy basins, which were labeled in the pictures. 

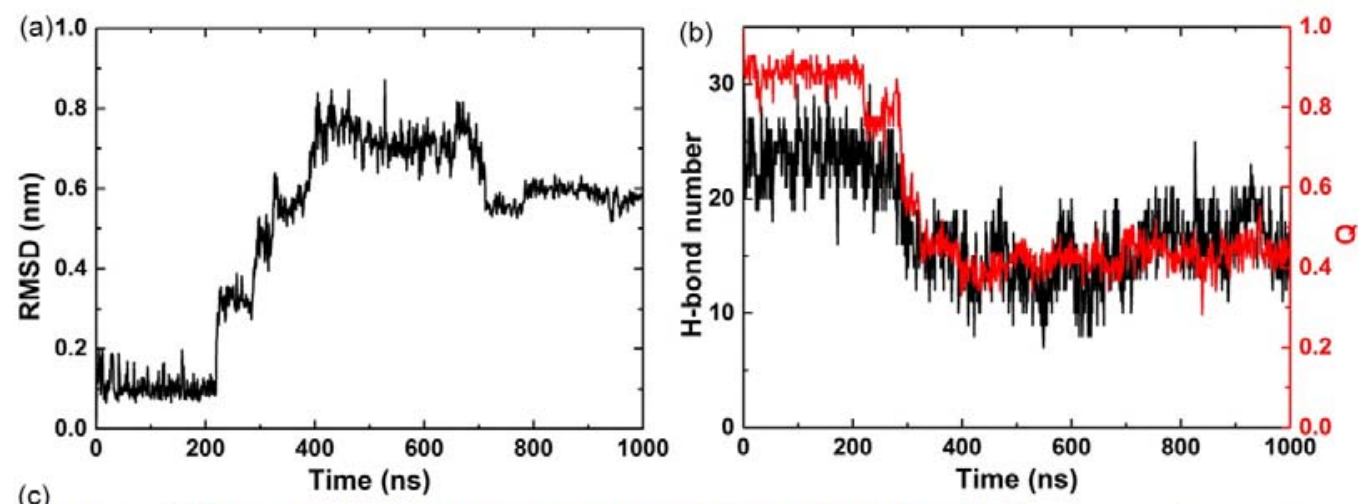

(c)

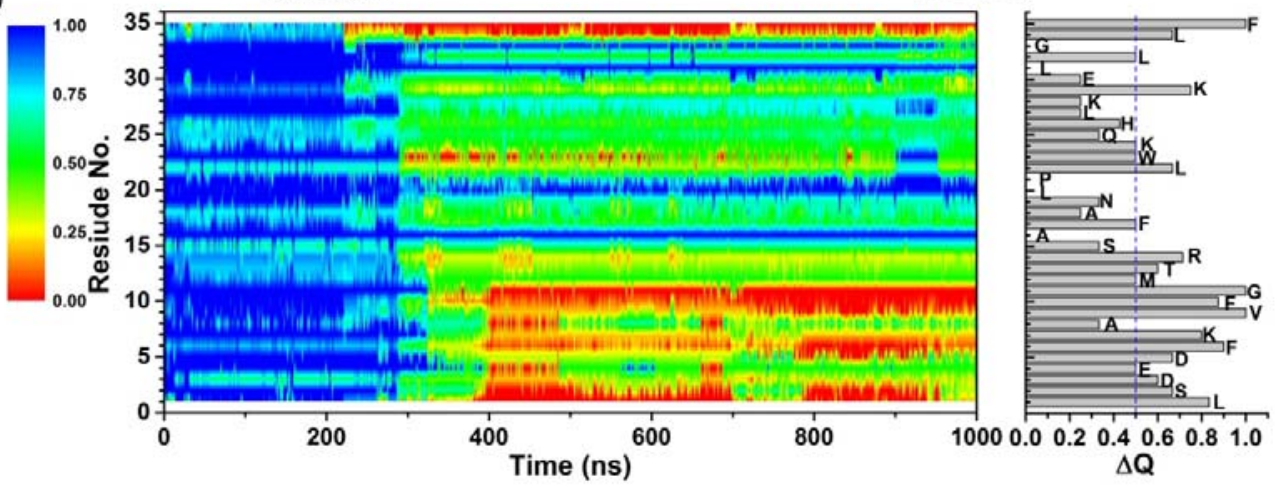

(d)

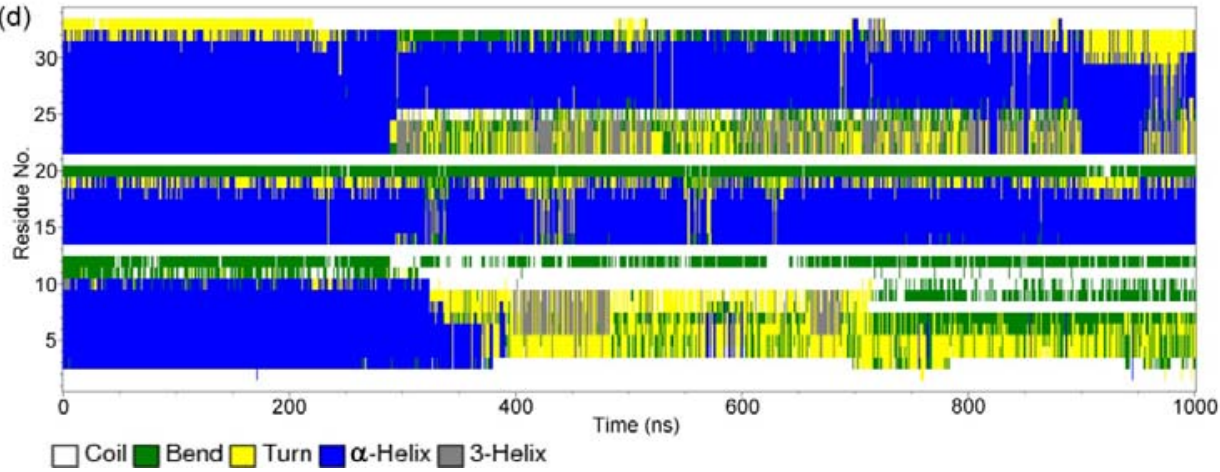

Figure 4. Structural change analyses from a typical trajectory. (a) Root mean square deviation

(RMSD) values of HP35 with respect to the simulation time. (b) Time-evolution hydrogen bond number and native contact $\mathrm{Q}$ were depicted in black and red, respectively. (c) Time-dependent $\mathrm{Q}$ values for each HP35 residue during the simulation (left) and the Q difference value between 0 ns and $1000 \mathrm{~ns}$ for each residue (right). (d) The secondary structure map versus the simulation time. 


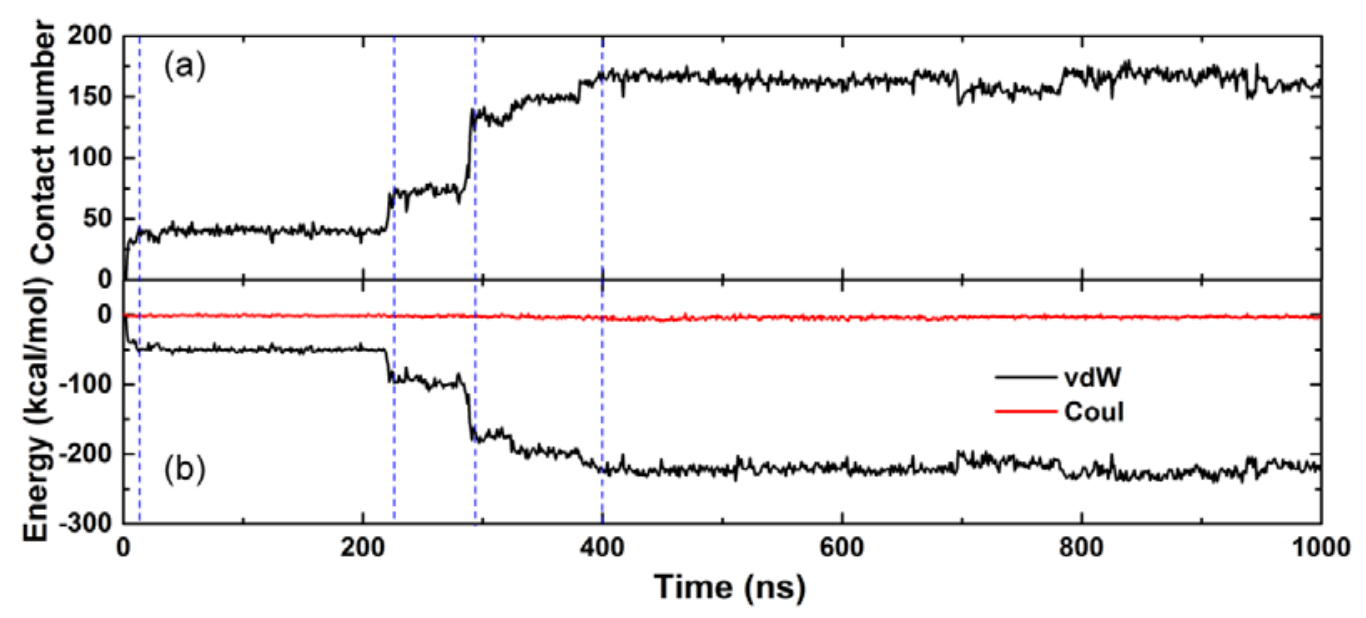

(c)
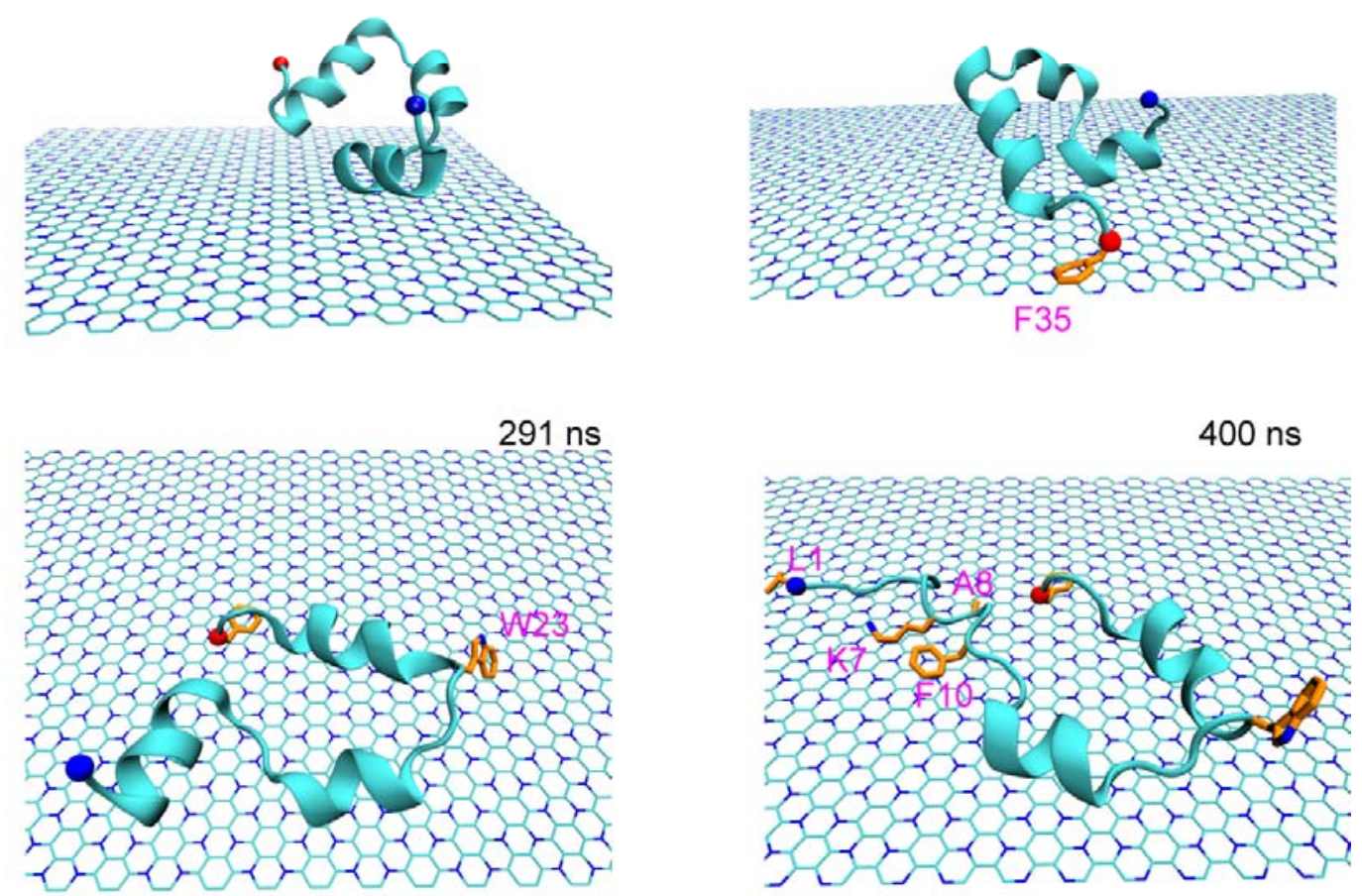

Figure 5. Kinetics of HP35 unfolding on $\mathbf{C}_{3} \mathbf{N}$ nanosheet. Time-evolution contact number (a) and interaction energies (including vdW and Coulomb contributions) of the HP35 absorption process to the $\mathrm{C}_{3} \mathrm{~N}$ nanosheet. Blue dashed lines indicated significant time points for the adsorption and unfolding events of HP35. (c) Key snapshots depicted the binding and unfolding processes. Some important residues described in the main text were also displayed. 


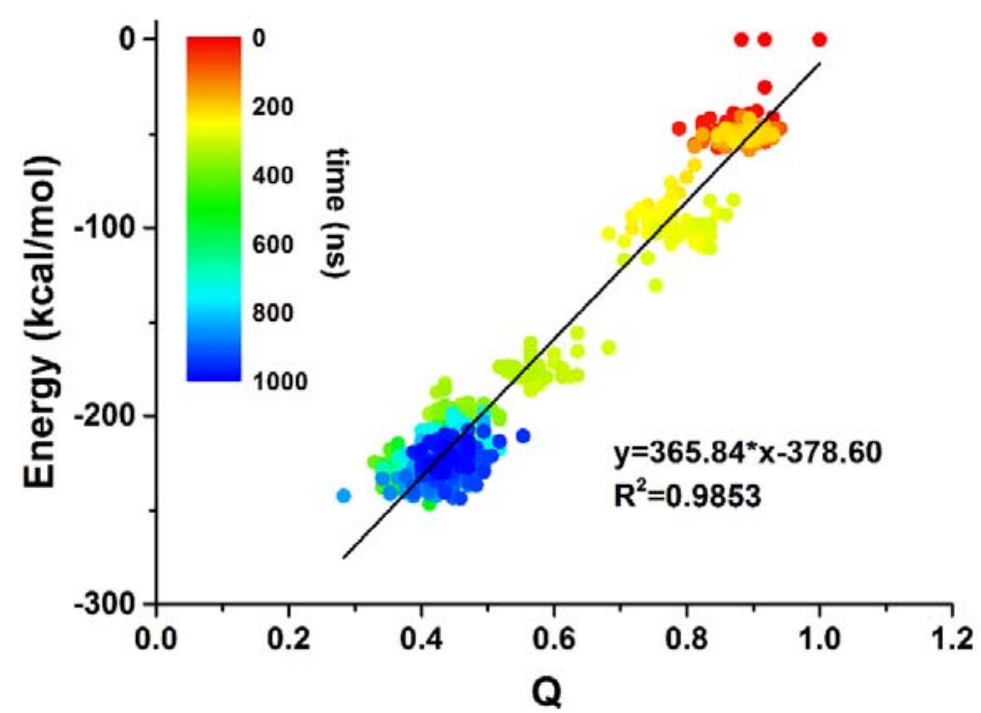

Figure 6. Time-dependent linear correlation of interaction energy and native $Q$ ratio. The color dots were collected every nanosecond (i.e., 1000 dots). 